\title{
Dnmt3a deletion cooperates with the FIt3/ITD mutation to drive leukemogenesis in a murine model
}

\author{
Jennifer L. Poitras ${ }^{1}$, Diane Heiser ${ }^{1}$, Li Li ${ }^{1}$, Bao Nguyen ${ }^{1}$, Kozo Nagai ${ }^{1}$, Amy S. \\ Duffield ${ }^{2}$, Christopher Gamper ${ }^{1,3}$ and Donald Small ${ }^{1,3}$ \\ ${ }^{1}$ Department of Oncology, Johns Hopkins University School of Medicine, Baltimore, MD, USA \\ 2 Department of Pathology, Johns Hopkins University School of Medicine, Baltimore, MD, USA \\ ${ }^{3}$ Department of Pediatrics, Johns Hopkins University School of Medicine, Baltimore, MD, USA \\ Correspondence to: Donald Small, email: donsmall@jhmi.edu
}

Keywords: FLT3, internal tandem duplication, DNMT3a, acute myeloid leukemia, mouse model

Received: May 20, $2016 \quad$ Accepted: September 02, $2016 \quad$ Published: September 12, 2016

\section{ABSTRACT}

Internal tandem duplications of the juxtamembrane domain of FLT3 (FLT3/ ITD) are among the most common mutations in Acute Myeloid Leukemia (AML). Resulting in constitutive activation of the kinase, FLT3/ITD portends a particularly poor prognosis, with reduced overall survival and increased rates of relapse. We previously generated a knock-in mouse, harboring an internal tandem duplication at the endogenous FIt3 locus, which develops a fatal myeloproliferative neoplasm (MPN), but fails to develop acute leukemia, suggesting additional mutations are necessary for transformation. To investigate the potential cooperativity of FLT3/ITD and mutant DNMT3A, we bred a conditional Dnmt3a knockout to a substrain of our FIt3/ITD knock-in mice, and found deletion of Dnmt3a significantly reduced median survival of $F / t 3^{I T D /+}$ mice in a dose dependent manner. As expected, pIpC treated $F / t 3^{I T D /+}$ mice solely developed MPN, while FIt $3^{I T D /+} ;$ Dnmt3af/f and FIt $3^{I T D /+} ; D n m t 3 a^{f /+}$ developed a spectrum of neoplasms, including MPN, T-ALL, and AML. Functionally, FLT3/ITD and DNMT3A deletion cooperate to expand LT-HSCs, which exhibit enhanced self-renewal in serial re-plating assays. These results illustrate that DNMT3A loss cooperates with FLT3/ITD to generate hematopoietic neoplasms, including AML. In combination with FLT3/ITD, homozygous Dnmt3a knock-out results in reduced time to disease onset, LT-HSC expansion, and a higher incidence of T-ALL compared with loss of just one allele. The co-occurrence of FLT3 and DNMT3A mutations in AML, as well as subsets of T-ALL, suggests the FIt $3^{I T D /+} ; D n m t 3 a^{f / f}$ model may serve as a valuable resource for delineating effective therapeutic strategies in two clinically relevant contexts.

\section{INTRODUCTION}

Large scale genomic studies have revealed the complex and heterogeneous genomic underpinnings of Acute Myeloid Leukemia (AML), identifying FLT3 as the most frequently mutated gene in the disease, occurring in $\sim 30 \%$ of AML patients.[1-3] Internal tandem duplication (ITD) of the juxtamembrane domain is the most common of these mutations, and predicts poor clinical outcomes.[3] Previous studies in knock-in mice illustrate that Flt $3 \mathrm{I}^{T D /+}$ alone generates a myeloproliferative neoplasm (MPN) and is insufficient to drive leukemogenesis, suggesting additional mutations are necessary for full transformation. $[4,5]$ Global genomic sequencing studies have identified a substantial subset of patients in which FLT3/ITD and DNMT3A mutations are concomitantly present.[1, 3] Moreover, the co-occurrence of these mutations is significantly associated with adverse clinical outcomes. [3] Typically in AML patients, sequencing studies have demonstrated 8-13 mutations but it is unclear how many of these mutations are drivers versus passengers, leaving in doubt the number of mutations required for full transformation of primary cells.[6] Based on these 
observations, investigating the potential cooperativity of these mutations in a murine model serves to answer important questions regarding the underlying biology of the disease, while serving as a powerful drug discovery tool with the potential for significant clinical impact.

DNMT3A mutations are among the most common alterations in AML, just behind FLT3/ITD.[1, 2, 7] While AML-associated mutations have been identified throughout the body of the gene, the overwhelming majority are heterozygous missense mutations within the catalytic domain, often affecting Arginine 882.[8] In vitro studies suggest the $\mathrm{R} 882 \mathrm{H}$ mutation leads to reduced methyltransferase activity, and acts in a dominant negative manner by impairing tetramer formation.[9-11]

In accordance with mounting evidence that DNMT3A mutations result in a loss of function, conditional knock-out mice have been used extensively to evaluate the effects of Dnmt3a loss on stem cell function and leukemia development.[12, 13] These mice harbor floxed Dnmt3a alleles $\left(D n m t 3 a^{f / f}\right)$ and Mx1-Cre, which is induced upon injection with polyinosinic-polycytidylic acid (pIpC).[14] Recent work demonstrated that upon serial transplantation, Dnmt3a ablation in hemaotpoietic stem cells (HSC) promotes self-renewal and expansion of the LT-HSC pool, with progressive differentiation and methylation defects of downstream progeny.[13, 15] As DNMT3A mutations are thought to represent one of the earliest founding events in AML, these functional studies may explain how mutant DNMT3A initiates the disease, through expanding the primitive cell pool, thereby increasing the probability of acquiring additional deleterious mutations.[16, 17] Conversely, Flt3/ITD disrupts LT-HSC quiescence, resulting in depletion of this compartment.[18] Given the co-occurrence of FLT3/ITD and DNMT3A mutations in AML patients, we hypothesized that Dnmt $3 a$ deletion may cooperate with Flt3/ITD to induce leukemia, potentially through rescuing the LT-HSC depletion seen in Flt $3^{I T D /+}$ mice.

Since $D N M T 3 A$ mutations seem to represent an initiating event, breeding Dnmt $3 a^{f / f}$ mice to our Flt $3^{I T D /+}$ knock-in mice presents a significant challenge, as mutant Flt3 is expressed at the earliest stages of hematopoeisis, preceding Dnmt3a deletion. To partially circumvent this issue, we used a substrain of our Flt $3^{I T D /+}$ knock-in mice which retains a floxed Neomycin $(\mathrm{Neo})$ selection cassette from the initial targeting (Flt $3^{\text {ITDneo/+}}$; referred to hereafter as Flt $3^{I T D /+}$ for simplicity's sake). The presence of the cassette greatly reduces expression of the mutant allele. [4] Flt $3^{I T D /+}$ expression is fully restored upon excision of the cassette following Cre induction, effectively "knocking in" the Flt3/ITD mutation and knocking out Dnmt3a simultaneously (Figure 1A). This novel approach allowed us to perform long term survival studies and disease characterization in a biologically relevant context.

\section{RESULTS}

\section{Dnmt3a deletion reduces survival of FIt $^{\mathrm{ITD} /+}$ knock-in mice in a dose dependent manner}

In accordance with mounting evidence that DNMT3A mutations result in a loss of function, we used a mouse model harboring floxed Dnmt $3 a$ alleles (Dnmt $3 a^{\text {tf }}$ ), and a Cre transgene under control of the type I interferon inducible Mx1 promoter (Mx1-Cre), which is activated upon injection with $\mathrm{pIpC}$, inducing LoxP recombination and excision in hematopoietic cells. $[9,14,19]$ These mice were bred to a substrain of our Flt $3^{I T D /+}$ knock-in mice, which retains a floxed PGK-Neo selection cassette from the initial targeting (Figure 1A). The presence of the cassette reduces transcription of the mutant allele, and full expression is restored upon Cre excision.[4] Since DNMT3A mutations are thought to represent one of the earliest events in leukemia development, this approach provides the unique advantage of temporally restraining Flt $3^{I T D}$ expression until Dnmt3a is lost; effectively "knocking in" the Flt3 ITD mutation, and knocking out Dnmt3a simultaneously upon pIpC injection.[2, 16, 17] Mx1-Cre expressing mice harboring Flt $3^{I T D /+}$ and homozygous floxed Dnmt $3 a$ alleles $\left(F l t 3^{I T D /+} ; D n m t 3 a^{f / /}\right)$, heterozygous $\left(F l t 3^{I T D /+} ; D n m t 3 a^{f /+}\right)$, or wild type alleles

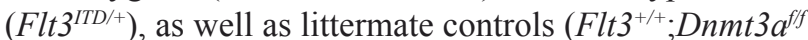
or Flt $3^{I T D /+} ;$ Dnmt $3 a^{f /+}$ lacking the Mx1-Cre transgene) were injected with $\mathrm{pIpC}$ intraperitoneally at 8 weeks of age and monitored for disease development. Peripheral blood was collected every 1-2 months after injection to monitor changes in the white blood cell differential, and to collect genomic DNA to confirm effective loxP recombination (Figure S1).

Interestingly, deletion of Dnmt3a significantly reduced median survival of Flt $3^{\text {ITD/+ }}$ mice in a dosedependent manner, with median survival of 162 days and 256 days for Flt $^{I T D /+} ;$ Dnmt $3 a^{f f /}$ and Flt $3^{I T D /+} ;$ Dnmt $3 a^{f /+}$, respectively, irrespective of disease diagnosis (Figure 1B). Both genotypes confer a significantly shorter survival time compared to Flt $3^{I T D /+}$ mice alone, which have a median survival of 412 days, consistent with our previous findings.[4, 20, 21] Dnmt3a dosage was also associated with a number of other parameters, including splenomegaly (Figure 1C) and leukocytosis (Figure 1D). While the trending increase in spleen weight and WBC is evident, only Flt $3^{I T D /+}$ mice with complete loss of Dnmt3a $\left(\right.$ Flt $^{I T D /+} ;$ Dnmt $\left.3 a^{f f}\right)$ displayed increases reaching statistical significance compared to Flt $3^{I T D /+}$ alone. 
Dnmt3a deletion cooperates with Flt3 $^{\text {ITD/+ }}$ to induce a broad spectrum of neoplasms including AML and T-ALL

As expected from previous work, mice with Flt $3^{I T D /+}$ alone developed MPN, while loss of one or both Dnmt3a alleles cooperates with the Flt $3^{I T D}$ mutation to elicit the development of an MPN or an acute leukemia of varying lineages including AML and T lymphoblastic leukemia / lymphoma (T-ALL) (Figure 2A). Histological analysis of bone marrow derived from mice with myeloid diseases revealed a predominance of myeloid blasts and immature myeloid cells in AML samples (Figure 2B). While T-ALLs displayed variable immunophenotypes, including double or single positive for CD4 or CD8 (data not shown), the immunophenotype among myeloid mice was fairly uniform, displaying an increase in blasts that are CD34+ and $\mathrm{Mac}^{+/ \mathrm{lo}}$, consistent with a myelomonocytic leukemia (Figure 2C, 2D).

In FLT3/ITD+ AML, loss of heterozygosity of the wild type allele is sometimes observed, and is associated with poorer survival.[22-24] To investigate whether or not this phenomenon also occurred in our model, genomic DNA was extracted from bone marrow and matched tail tissue from leukemic mice, and PCR amplification was performed using primers flanking the ITD mutation (Figure 3A). Loss of the wild type allele was observed in $36 \%(5 / 14)$ of mice developing AML (both Flt $3^{I T D /+} ;$ Dnmt $3 a^{f f}$ and $F l t 3^{I T D /+} ;$ Dnmt $3 a^{f /+}$ ), while no evidence of complete loss of wild-type allele was observed in any of the 13 mice developing lymphoid

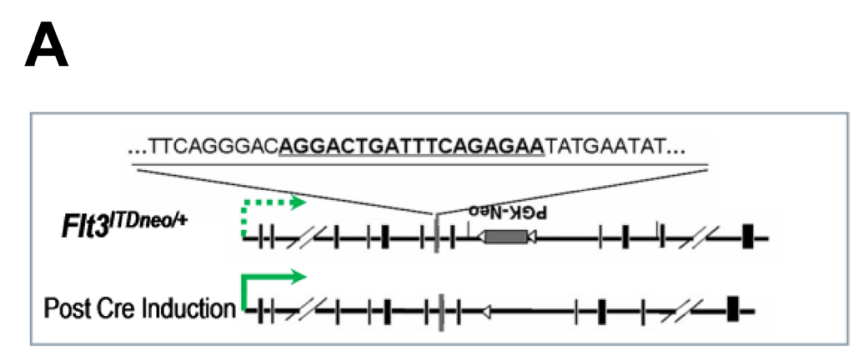

B
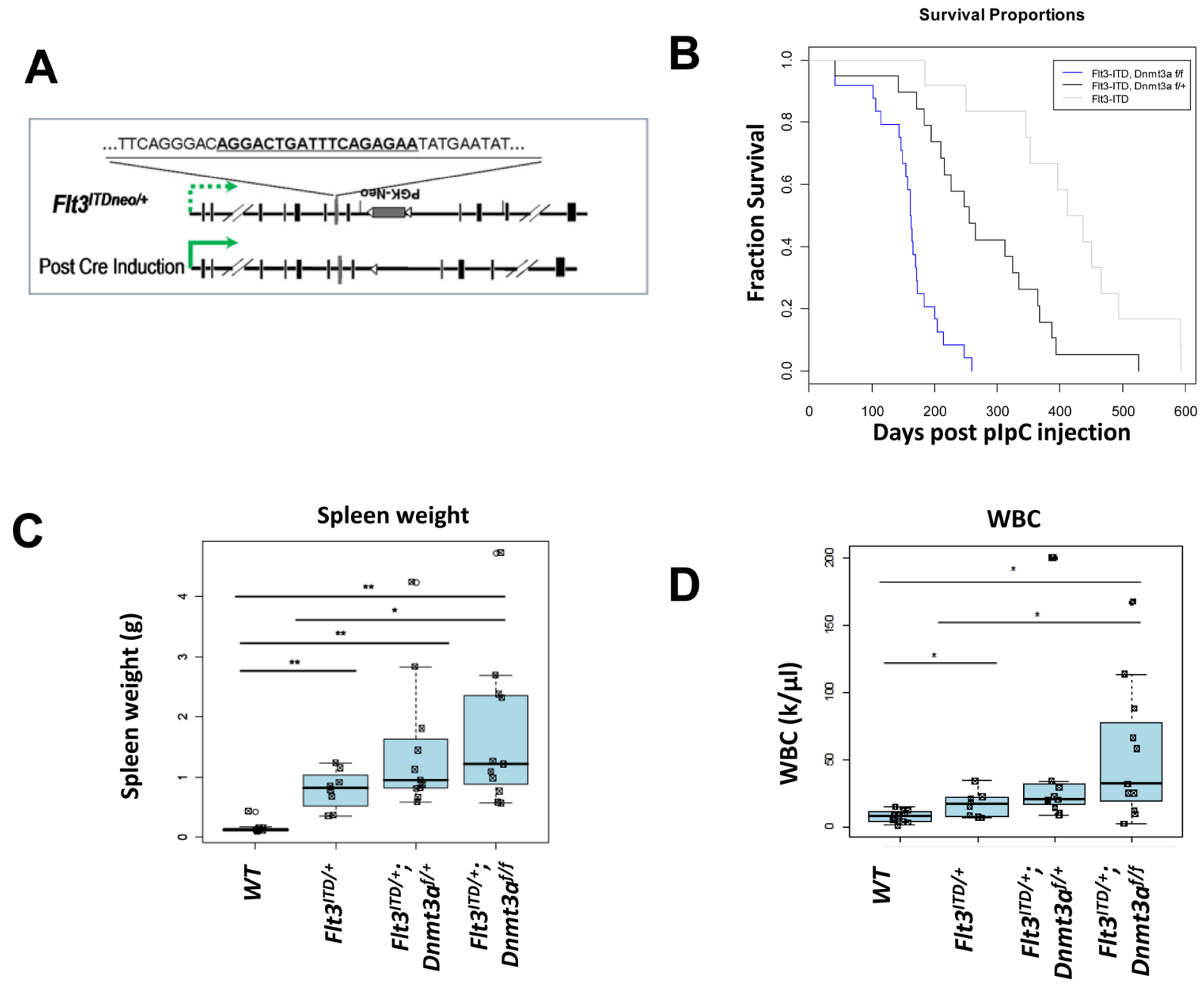

Figure 1: Dnmt3a deletion cooperates with Flt $^{I T D /+}$ to shorten survival in a dose dependent manner. A. Strategy for temporally controlling Flt3/ITD expression using a substrain of our Flt $3^{I T D /+}$ knock-in mice. The substrain $\left(\right.$ Flt $3^{\text {ITDneo/+ }}$ ) retains the PGK-Neo cassette inserted at the mutant allele (referred to hereafter as $F l t 3^{I T D /+}$ ). The presence of the cassette reduces expression of the mutant allele (dashed green arrow), and $F l t 3^{I T D /+}$ expression is fully restored upon excision of the cassette following cre induction (solid green arrow). B. Kaplan-Meier Survival Curve. Median survival is 162 days for Flt $3^{I T D /+} ; D n m t 3 a^{f f /}(n=24)$, 260 days for Flt $3^{I T D /+} ; D n m t 3 a^{f /+}(n=20)$, and 412 days for $F l t 3^{I T D /+}$ alone $(n=12)$. C. Spleen weights, and D. White blood cell counts $(\mathrm{WBC})$ of mice developing myeloid neoplasms at the time of sacrifice. Wildtype (WT), FLT3/ITD alone $\left(F l t 3^{I T D /+}\right), F l t 3^{I T D /+} ; D n m t 3 a^{f /+}, F l t 3^{I T D /+} ;$ Dnmt $3 a^{f f} .\left(^{*}, p<0.05 ; * *, p<0.01\right)$. 


\section{A}

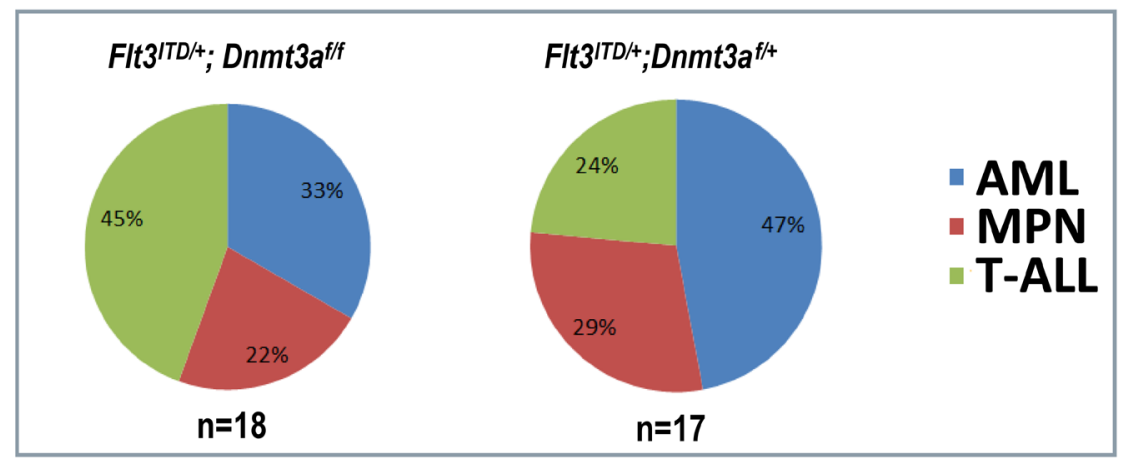

B
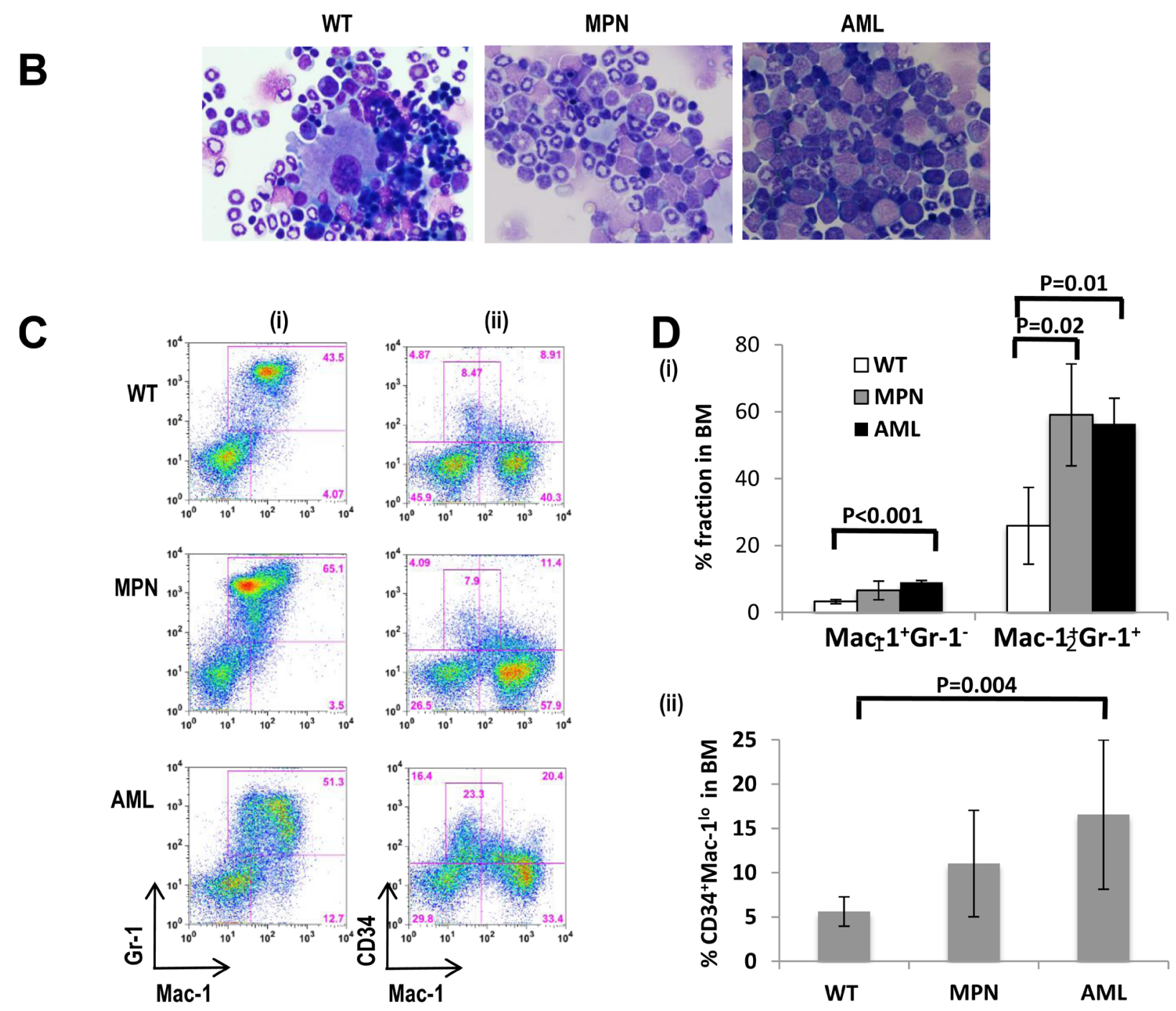

Figure 2: Dnmt3a deletion cooperates with $\mathrm{Flt}^{I T D /+}$ to induce a broad spectrum of neoplasms. A. Disease distribution of Flt $3^{I T D /+}$ mice lacking both (left) or a single (right) Dnmt3a allele. B. Hematoxylin and Eosin stained bone marrow cytospins from a representative wild type mouse (WT, left column), a mouse with a Myeloproliferative Neoplasm (MPN, center column), and a mouse with Acute Myeloid Leukemia (AML, right column). C., D. Flow cytometric analysis of bone marrow. (i) The MPN mouse displays the typical $\mathrm{Mac}^{+} \mathrm{Grl}^{+}$expansion, while the leukemic mouse displays an abnormal $\mathrm{Macl}^{+} \mathrm{Gr}^{10 /}$ population. (ii) The wild-type mouse displays the normal CD34 positivity, while AMLs exhibit increased immature $\mathrm{CD} 34^{+}$cells which appears to be Mac ${ }^{10} \mathrm{CD} 34^{+}$. In this experiment, $n=$ 5 mice for each group. 
neoplasms and the 8 mice developing myeloproliferative neoplasms (Figure 3B). None of the $8 \mathrm{MPN}$ BM samples showed complete loss of the wild-type allele. However, it is notable that 3 of the MPN BM samples exhibited a higher ITD $v s$ wild-type allelic ratio. This might indicate that loss of wild-type allele is occurring in some but not all of the BM cells, perhaps on their way to developing AML (Figure 3B). These findings are consistent with previous studies in Flt $3^{I T D /+}$ knock-in mice, where loss of the wild type allele is restricted to AML, and absent in T-ALL, suggesting a selective advantage for these events specifically in the myeloid lineage. $[20,25]$

\section{T-ALL and AML samples are transplantable, with transplanted T-ALL exhibiting particularly aggressive disease}

To investigate transplantability of primary neoplasms developing in our mice, cells from each leukemic donor were transplanted into three sublethally irradiated recipients and monitored for survival (Figure 3C). Engraftment and survival were variable based on disease subtype, as all 4 of the AML samples from Flt $3^{I T D /+} ;$ Dnmt3 $a^{f / f}$ donors engrafted, with a median survival of 174 days, while only 2 of $4 F l t 3^{\text {ITD/+ }}$;Dnmt $3 a^{f / 4}$ AML samples engrafted, displaying slightly prolonged (but not statistically significant) median survival. Transplantation of MPNs derived from Flt $3^{I T D /+}$ mice failed to engraft, consistent with previous findings.[4] In stark contrast, T-ALL samples from both Flt $3^{I T D /+} ; D n m t 3 a^{f f f}$ and Flt $3^{I T D /+} ; D n m t 3 a^{f /+}$ genotypes were much more aggressive, killing recipients within one month.

\section{T-ALLs in mice with FLT3-ITD and DNMT3A deletion exhibit repressed Fit3 expression}

Previous sequencing endeavors have identified DNMT3A and FLT3 as frequently mutated genes in AML, both individually and concurrently, and at lower frequencies in T-ALL.[26-28] While lymphoid leukemias haven't previously been observed in our Flt $3^{I T D /+}$ knock-in model, previous work has demonstrated that $\sim 15 \%$ of Dnmt $3 a^{f / f}$ mice develop T-ALL, with a median survival of 246 days, compared with 162 days in our Flt $3^{I T D /+}$;Dnmt3 $a^{f f f}$ mice.[12] Taken together, these findings suggest that perhaps the Flt3/ITD mutation is dispensable for maintenance of lymphoid malignancies in $F l t 3^{I T D /+} ; D n m t 3 a^{f / f}$ mice, but may play an important
A

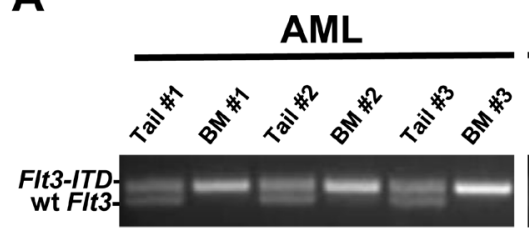

C

\begin{tabular}{|c|c|c|c|c|}
\hline Genotype & Disease & \#Recipients & \#Engrafted & $\begin{array}{l}\text { Median Survival } \\
\text { (days) }\end{array}$ \\
\hline FIt3/TD/+;Dnmt3 $a^{f / f}$ & AML & 12 & 12 & 174 \\
\hline$F / t 3^{I T D /+} ; D n m t 3 a^{f / t}$ & AML & 12 & 6 & 210 \\
\hline Flt3 $3^{I T D /+} ;$ Dnmt3a $a^{+/+}$ & MPN & 6 & 0 & N/A \\
\hline Flt3 $3^{I T D /+} ; D n m t 3 a^{f / f}$ & T-ALL & 6 & 6 & 29 \\
\hline Flt3/TD/+;Dnmt3a ${ }^{f /+}$ & T-ALL & 3 & 3 & 16 \\
\hline
\end{tabular}

B

\begin{tabular}{|c|c|c|}
\hline Genotype & Disease & Fraction LOH \\
\hline FIt $3^{I T D /+} ;$ Dnmt3af/+ or $f / f$ & AML & $5 / 14$ \\
\hline$F / t 3^{I T D /+} ; D n m t 3 a^{f /+}$ or $f / f$ & MPN & $0 / 8$ \\
\hline Flt3 ${ }^{I T D} /+; D n m t 3 a^{f /+}$ or $f / f$ & T-ALL & $0 / 13$ \\
\hline
\end{tabular}

D

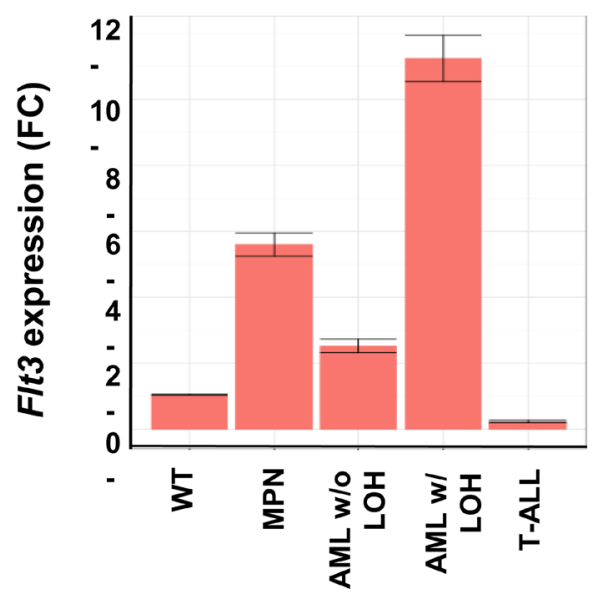

Figure 3: Loss of Heterozygosity (LOH) and Flt3 expression are present in a disease specific manner. A. Representative samples exhibiting bone marrow specific LOH at the Flt3 locus. Primers flanking the ITD mutation were used to amplify genomic DNA prepared from bone marrow and matched tail tissue from representative AML mice (\#1, \#2 and \#3) and MPN mice (\#4 and \#5). B. Summary of samples exhibiting LOH. Complete loss of the wild type Flt3 allele occurs exclusively in mice developing AML, in both Flt $3^{I T D /+}$;Dnmt $3 a^{f f}$ and $F l t 3^{I T D /+} ; D n m t 3 a^{f /+}$ genotypes. BM from some of the MPN mice demonstrated higher ITD vs. wt allelic ratio but no signs of complete loss of the wild-type allele. C. Survival summary of transplantation experiments. Diseases of donors and median survival of all engrafted recipients are noted. D. T-ALLs are driven by FLT3/ITD-independent factors. qPCR of leukemic tissue displays silencing of Flt 3 in lymphocytic leukemia samples. RNA was extracted from whole bone marrow of Wildtype (WT), Flt $3^{I T D /+} ;$ Dnmt $3 a^{f f f}$ mice developing MPN, AML without LOH, AML with LOH at the Flt3 locus, or T-ALL. $N=5$ mice per genotype in each experiment. 
role in expanding the progenitor pool giving rise to these lymphoid neoplasms, thereby accelerating disease development.

To examine Flt3 expression, we performed qPCR on whole bone marrow from wild type and Flt $3^{I T D /+} ;$ Dnmt $3 a^{f / f}$ mice with varying diagnoses. Flt3 expression was elevated in myeloid neoplastic samples, especially those exhibiting $\mathrm{LOH}$, with expression levels at about 11 fold higher than wild type bone marrow samples (Figure 3D). Interestingly, T-ALL samples expressed virtually no Flt3, supporting the hypothesis that once full transformation occurs in these cells, Flt3 is turned off, as in normal lymphoid development.[29]

\section{Myeloid progenitors are expanded in double mutant mice at an early time point, underlying observed relative monocytosis and disease variability}

Recent work has demonstrated that Dnmt3a deletion in HSC, promotes self-renewal and expansion of the
A

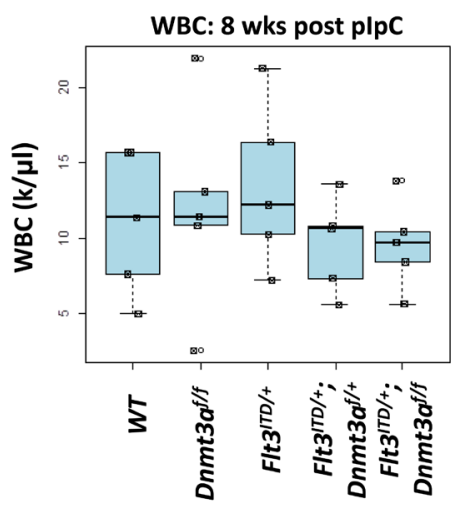

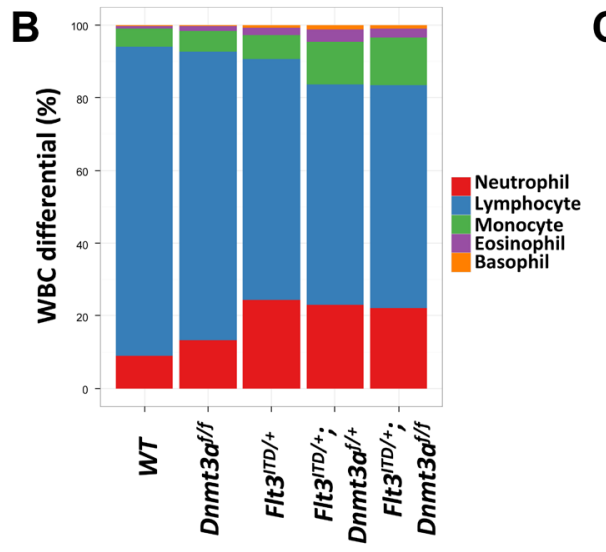

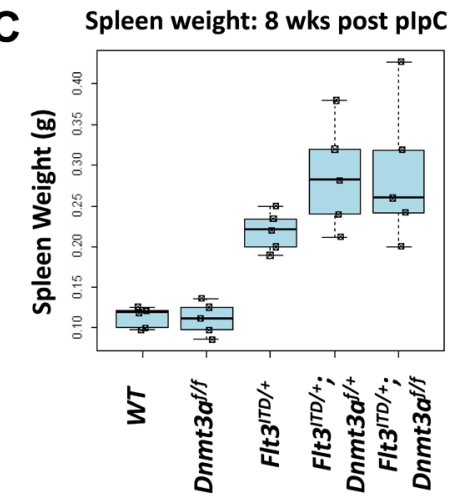

D

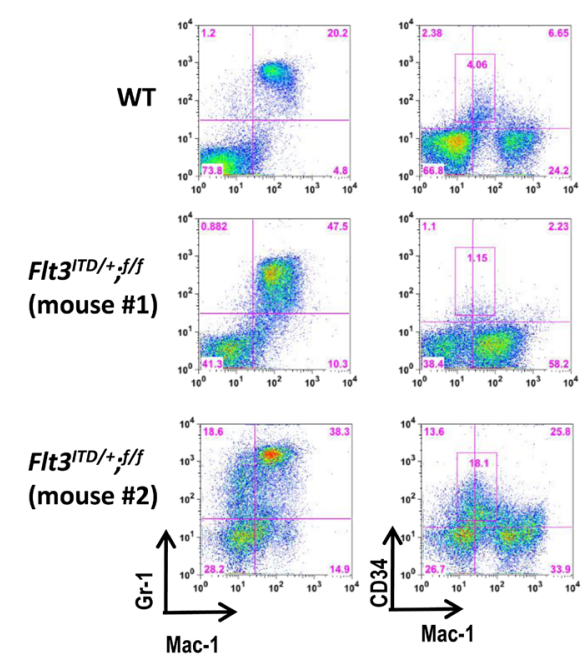

E

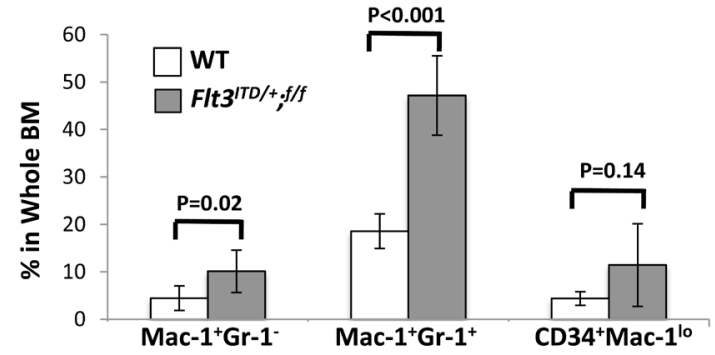

$\mathbf{F}$

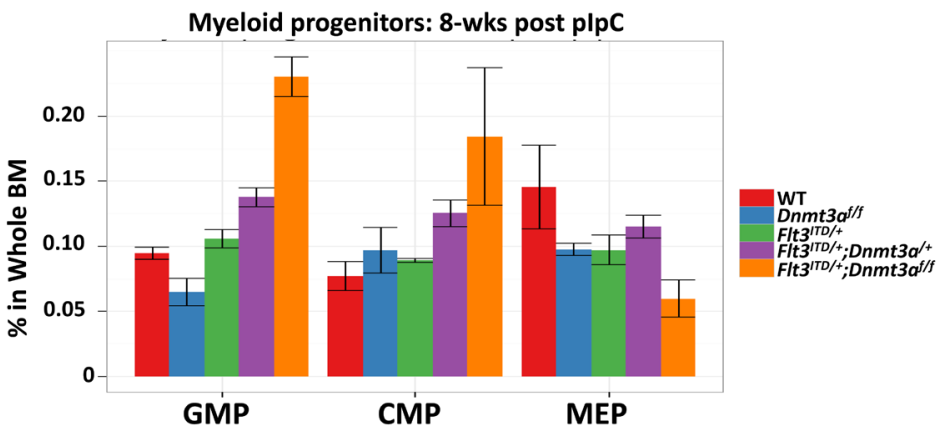

Figure 4: Early signs of disease development and myeloid expansion are present at 8 weeks post pIpC injection. A. WBC remain within the normal range for all genotypes. B. Relative monocytosis is observed upon CBC, despite normal WBC. WT and $D n m t 3 a^{f f f}$ exhibit normal leukocyte ratios, while $F l t 3^{I T D /+}$ mice display an increase in percentage of neutrophils and concomitant decrease in percentage of lymphocytes. When these mice lose one or both Dnmt3a alleles, the phenotype is exaggerated with further decrease in relative lymphocytes and an increase in the percentage of monocytes. C. Spleen weight is increased only in mice harboring a FLT3/ITD mutation. Splenomegaly is further enhanced with loss of one or both Dnmt3a alleles. D., E. Flow cytometric analysis of bone marrow at 8 weeks reveals abnormal patterns of myeloid maturation and CD34 expression. Two representative mutant mice are shown in D. $N=5$ and 10 for the wild-type and $F l t 3^{I T D /+} ; D n m t 3 a^{f f f}$ groups, respectively. F. Shifts in the myeloid progenitor compartment are present. Wildtype (WT), Dnmt $3 a$ knockout alone $\left(\right.$ Dnmt $\left.3 a^{\mathrm{f} / \mathrm{f}}\right)$, FLT3/ITD alone $\left(F l t 3^{I T D /+}\right)$, Flt $3^{I T D /+} ;$ Dnmt $3 a^{f /+}$ and $F l t 3^{I T D /+} ;$ Dnmt $3 a^{f / f}\left(F l t 3^{I T D /+} ; f f /\right)$. $N=5$ mice per genotype in each experiment. 
LT-HSC pool, resulting in leukemia with incomplete penetrance and prolonged time to disease development. Conversely, Flt $3^{I T D /+}$ disrupts LT-HSC quiescence, resulting in depletion of this compartment, and failure to develop acute leukemia in the absence of cooperating mutations. [12, 13, 18] We hypothesized that enhanced self-renewal and expansion in the LT-HSC compartment conferred by Dnmt3a deletion might "rescue" the LTHSC depletion seen in Flt $3^{I T D /+}$ mice, thereby increasing this primitive pool and the opportunity for additional mutations necessary to drive either myeloid or $\mathrm{T}$ cell leukemia to develop. To investigate the frequencies of progenitor populations well prior to disease onset, mice were sacrificed at 8 weeks post $\mathrm{pIpC}$ injection and the stem cell compartment was examined by flow cytometry.

While overt leukemia was absent at this early time point, pathological changes were evident in the bone marrow and peripheral blood. None of the genotypes exhibited leukocytosis (Figure 4A), but relatively increased fractions of granulocytes and monocytes were seen, as expected, in Flt $3^{I T D /+}$ mice, and was exaggerated in the Flt $3^{I T D /+} ;$ Dnmt $3 a^{f /+}$ and Flt $3^{I T D /+} ;$ Dnmt $3 a^{f f f}$ mice, with a concomitant decrease in percent lymphocytes (Figure 4B). While Dnmt $3 a$ ablation alone had no effect on spleen size at 8 weeks, splenomegaly was noted in Flt $3^{I T D /+}$ mice, which was again more pronounced in double mutant mice (Figure 4C). Flow cytometric analysis of whole bone marrow displayed varying anomalies, including aberrant patterns of myeloid antigen and CD34 expression as well as expansion within the myeloid lineage (Figure 4D). This immunophenotypic variability at an early time point likely underlies the ultimate variability in neoplasms developing in moribund mice. Interestingly, while percentage shifts favoring myeloid cell types in the peripheral blood were

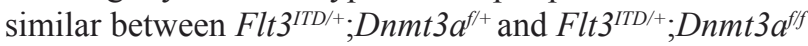
mice, appreciable shifts in myeloid progenitor populations were only present in the Flt $3^{I T D /+}$;Dnmt $3 a^{f / f}$ bone marrow samples, where obvious increases in common myeloid (CMP) and granulocyte-macrophage (GMP) progenitors were observed with a decrease in megakaryocyteerythrocyte progenitors (MEP) (Figure 4F).
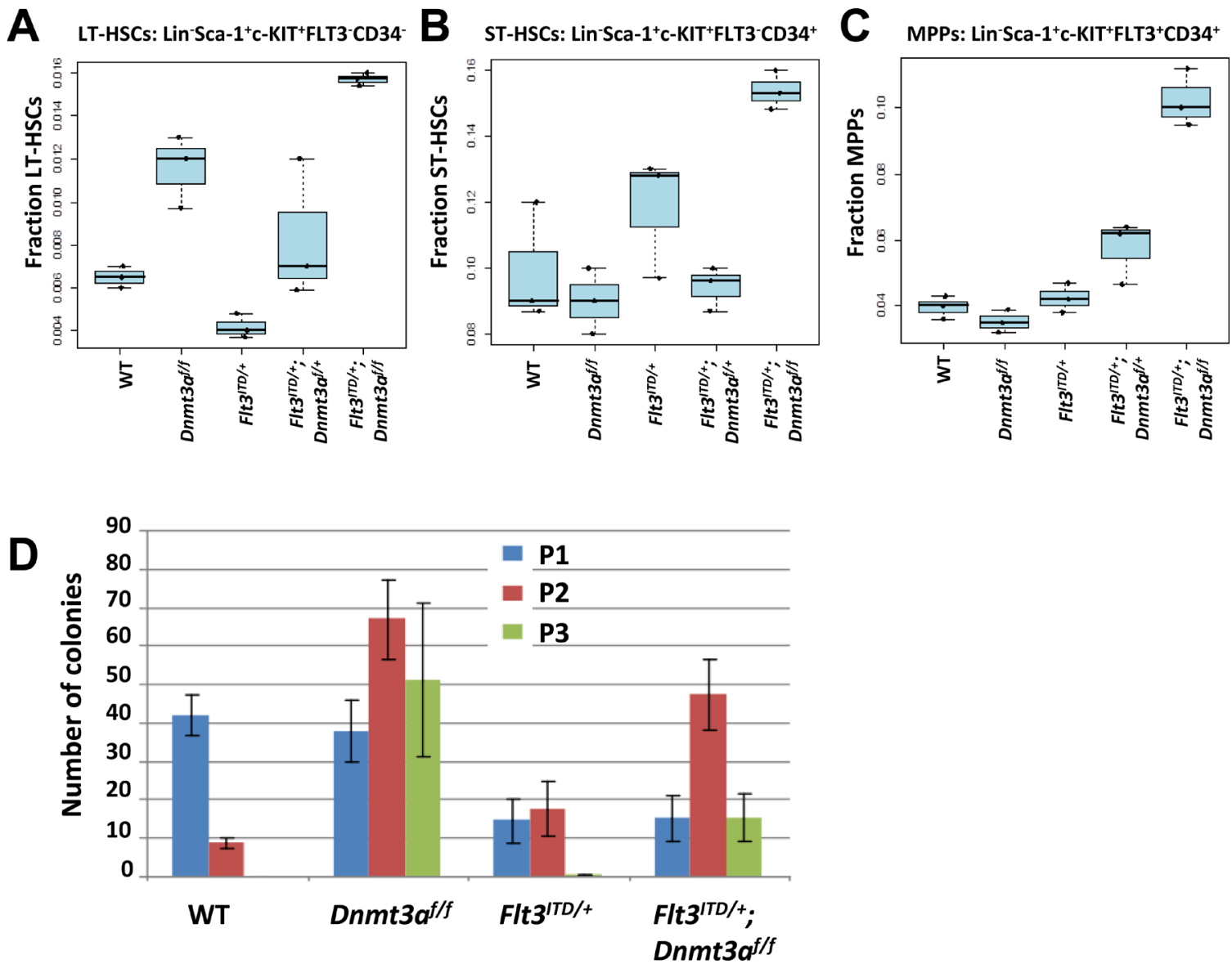

Figure 5: Dnmt3a deletion rescues the LT-HSC depletion phenotype seen in Flt $^{I T D /+}$ knock-in mice. A. LT-HSC, B. STHSC, and C. MPP populations were quantified by flow cytometric analysis of whole bone marrow of mice at 8 weeks post pIpC. $n=3$ per genotype. D. Serial replating of Lineage negative bone marrow at 8 weeks post pIpC from 3 mice per genotype. Each plating was performed in triplicate. Wildtype (WT), Dnmt $3 a$ knockout alone $\left(D n m t 3 a^{\mathrm{flf}}\right)$, FLT3/ITD alone $\left(F l t 3^{I T D /+}\right), F l t 3^{I T D /+} ; D n m t 3 a^{f /+}$ and $F l t 3^{I T D /+} ; D^{2} m t 3 a^{f / f}$. 


\section{Loss of both copies of Dnmt3a is necessary to elicit LT-HSC expansion}

Upon examining the stem cell compartment, we confirmed the previously described expansion in Dnmt $3 a$ knock-out mice and depletion in Flt $3^{I T D /+}$ mice. The data suggest that loss of one Dnmt3a allele is sufficient to restore the LT-HSC $\left(\mathrm{Lin}^{-} \mathrm{c}-\mathrm{kit}^{+} \mathrm{Sca}-1^{+} \mathrm{Flt} 3^{-} \mathrm{CD} 34^{-}\right)$pool of Flt $3^{I T D /+}$ mice to wild type proportions, while loss of both Dnmt $3 a$ alleles together with Flt3/ITD expression results in a dramatic expansion, beyond that of Dnmt $3 a^{\text {fff }}$ alone (Figure5A). Given the previous findings that Dnmt3a deletion expands the LT-HSC compartment through enhanced quiescence, the observed minor decrease in the immediate downstream ST-HSC $\left(\mathrm{Lin}^{-} \mathrm{c}^{-\mathrm{kit}^{+}} \mathrm{Sca}-\mathrm{H}^{+}\right.$ $\left.\mathrm{Flt3}^{-} \mathrm{CD} 34^{+}\right)$(Figure 5B) and MPP $\left(\mathrm{Lin}^{-} \mathrm{c}^{-\mathrm{kit}^{+}} \mathrm{Sca}-1^{+}\right.$ Flt3 $3^{+} \mathrm{CD} 34^{+}$) (Figure 5C) populations in Dnmt3 $a^{\text {fff }}$ mice is not surprising. Likewise, as aberrant exit from quiescence driven by $\mathrm{Flt}^{I T D /+}$ alone resulted in LT-HSC depletion, these cells are driven to mobilize and differentiate, as evidenced by an observed increase in the ST-HSC (Figure 5B) and MPP (Figure 5C) pools. Similar to the LT-HSCs,

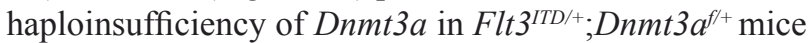
was sufficient to bring the fraction of ST-HSCs back towards the wild-type levels. In contrast, loss of both Dnmt $3 a$ alleles in Flt $3^{I T D /+} ;$ Dnmt $3 a^{f f f}$ mice resulted in a dramatic increase in the ST-HSC compartment (Figure 5B). Dnmt3a deletion in the Flt ${ }^{I T D /+}$ context conferred an increase in the MPP population in a dose dependent manner (Figure 5C).

\section{Dnmt3a ablation enhances colony formation of FIt $^{\mathrm{ITD} /+}$ bone marrow in serial replating experiments}

After determining that Dnmt3a loss and the Flt3/ ITD mutation do indeed cooperate to greatly expand the LT-HSC pool, we sought to investigate the mechanism underlying this observation. To assess self-renewal, lineage negative bone marrow cells were plated on methylcellulose, and serially re-plated twice more. The number of colonies was recorded before each re-plating. Since the methylcellulose used in these experiments contains cytokines directing myeloid differentiation, persistence of colony forming cells in the third plating suggests a block in differentiation and enhanced selfrenewal. As expected, wild type bone marrow displays a significant decrease in colony formation at the secondary plating and an absence of colonies at the tertiary re-plating. In agreement with previous observations, Dnmt $3 a^{f f 5}$ cells continued to re-plate through the tertiary plating, exhibiting enhanced self-renewal. Only one tertiary replating colony was observed of all plates analyzed from Flt $3^{I T D / 4}$ mice, congruent with disrupted quiescence (Figure 5D). However, when Dnmt3a loss was added to the
Flt $3^{I T D /+}$ background, partial restoration of the enhanced self-renewal phenotype is observed (Figure 5D). These experiments suggest that the expansion in the LT-HSC pool of $F l t 3^{I T D /+} ;$ Dnmt $3 a^{f f f}$ mice is achieved, at least in part, by enhanced self-renewal conferred by Dnmt3a deletion.

\section{DISCUSSION}

Throughout the last decade, thorough analysis of the leukemia genome has generated an extensive compendium of AML-associated mutations. $[1,2]$ These data have formed the basis for subsequent functional studies in mice, using knock in, knock out, or transgenic strains. Mutation of the orthologous mouse gene represents the most biologically relevant experimental approach, yet many of the most common driver mutations do not, in isolation, lead to fully penetrant, rapidly fatal leukemias. $[4,5,21$, 30-32] These results are not surprising, as elegant studies dissecting the clonal evolution of leukemia indicate several mutations occur in concert, often chronologically, and are likely necessary for transformation.

Integrative genomic profiling has identified a significant proportion of AML patients with concomitant FLT3-ITD and DNMT3A mutations. Concurrently, these mutations stratify patients into a poorer prognostic category, predicting inferior clinical outcomes and overall survival.[3] It appears that neither FLT3/ITD nor DNMT3a mutations alone is sufficient for leukemic transformation. FLT3/ITD mutations have been shown to be a later event in leukemic clonal revolution. [33, 34] In contrast, loss of DNMT3a is one of the pre-leukemic events found in AML patients.[17, 34] In mouse, DNMT3A is highly expressed in HSCs enabling efficient differentiation through the epigenetic silencing of HSC regulatory genes.[13] Loss of DNMT3a promotes HSC self-renewal at the expense of differentiation. It is likely that the presence of the FLT3/ ITD and DNMT3a mutations provides mouse HSCs with self-renewal and proliferative advantages. At the same time, hematopoietic differentiation is blocked as a result of aberrant methylation of HSC regulatory genes caused by DNMT3a loss.

While Flt3/ITD knock-in alone fails to fully transform and recapitulate human leukemia, ablation of Dnmt3a alone is sufficient to predispose HSCs to malignant transformation, resulting in a spectrum of neoplasms with a prolonged time to disease development. [12] We hypothesized that breeding Flt $3^{I T D /+}$ mice and the conditional Dnmt3a knock-out would result in shortened survival compared to either mutation alone, cooperating to drive AML development in a greater proportion of mice than Dnmt $3 a^{f f f}$ alone.

It has recently been reported that the Flt $3^{\text {ITD/TTD }}$; $D n m t 3 a^{\text {fff }}$ mice spontaneously developed a fully penetrant AML while Flt $3^{\mathrm{ITD} /+} ;$ Dnmt $3 a$ knockout mice had a much longer disease latency and disease spectrum.[35] This suggests that the relative allelic ratio of ITD $v s$. wild type 
FLT3 is an important factor when evaluating the disease aggressiveness in the FLT3/ITD DNMT3a knockout double-mutant mice. Likewise, our data reported here confirm that indeed, Flt $3^{I T D /+}$ and bone marrow specific Dnmt3a deletion cooperate to result in shortened survival due to the development of fatal hematopoietic neoplasms, including AML and T-ALL. This is consistent with the recent finding of the development of AML and T-ALL in a similar FLT3/ITD and DNMT3 $a$-KO double-mutant mouse model.[36] Interestingly, we also found that Dnmt3a dosage significantly impacts survival and the spectrum of neoplasms developing in Flt $3^{I T D /+}$ mice. The observation that loss of a single allele is sufficient to shorten survival and elicit leukemia development highlights the importance of Dnmt3a stoichiometry in maintaining appropriate hematopoietic stem cell function. This finding is especially relevant in light of a recent meta-analysis of the TCGA cohort, which revealed a significant focal loss of $\mathrm{CpG}$ methylation throughout the genomes of AML patients harboring DNMT3A mutations.[9] Mean beta values were also further reduced in DNMT3A ${ }^{\mathrm{R} 882}$ compared to DNMT3A $\mathrm{A}^{\text {non-R882 }}$ samples, supporting in vitro evidence that R882 mutations impair methyltransferase activity more severely than mutations at other residues. Future assessment and comparison of these Flt $3^{I T D /+} ;$ Dnmt $3 a^{f /+}$ and $F l t 3^{I T D /+} ;$ Dnmt $3 a^{f / f}$ mice with human FLT3 $^{\text {ITD }}$;DNMT3 ${ }^{\text {non-R882 }}$ and FLT3 ${ }^{\text {ITD }}$;DNMT3A ${ }^{\text {R882 }}$ AML methylomes may reveal differentially methylated loci that are dosage, rather than sequence context dependent.

Unlike its high frequency in AML, FLT3/ITD mutation is a rare event in human T-ALLs, with an incidence of $2 \%$ or less and no prognostic significance. $[27,37]$ On the other hand, DNMT3a mutations occur frequently in adult T-ALL, ranging from $16 \%$ to $26.3 \%$ and are associated with poor prognosis and short survival.[8, 27, 38] Also unlike AML, which is characterized by heterozygous DNMT3A mutations primarily in the methyltransferase domain, the gene is frequently biallelically inactivated in T-ALL, with mutations occurring throughout the body of the gene. [8] Homozygous ablation of Dnmt3a in our mice may mimic biallelic inactivation seen in patients, accounting for the incidence of T-ALL in these mice. While Flt3 is virtually unexpressed in T-ALL derived from our double mutant mice, this does not preclude the possibility that FLT3/ITD is important for expansion of a lymphocytic progenitor, with cooperating somatic mutations and epigenetic changes favoring transformation within a later compartment, where FLT3 activity is dispensable.[29] The identification of FLT3 mutations in a subset of T-ALL patients raises important questions that warrant further investigation.[26, 27]

Variance in phenotype and delay in disease development may indicate that cells in the context of both mutations are still predisposed to transformation, but require additional mutations. Although leukemic mice meet the diagnostic criteria consistent with AML, including $>20 \%$ blasts, inconsistent engraftment makes it difficult to perform transplantation experiments and in vivo drug treatments. A recent study in AML1-ETO driven murine leukemia illustrates the utility and power of cross referencing genomic data from murine studies with the enormous repository of publicly available data from human leukemia samples sharing the same genetic underpinnings.[33] Using a similar approach in our model, we hope to identify cooperative orthologous genomic events which we can introduce into Flt $3^{I T D /+} ; D_{n m t 3} a^{f f /}$ bone marrow, thereby creating an increasingly relevant model of the disease, increasing aggressiveness and facilitating in vivo TKI and epigenetic combination therapy testing.

This model also presents a platform to answer important questions regarding DNMT3A dosage in the context of FLT3/ITD. Prior to leukemia formation, the incremental expansion of the LT-HSC pool indicates that differentially methylated loci important in maintaining quiescence can be determined. The data presented in this report demonstrate that conditional deletion of Dnmt3a and simultaneous "knock in" of Flt $3^{I T D /+}$, cooperate to drive leukemia development at a faster rate than Dnmt3a loss alone. Loss of heterozygosity of the Flt 3 allele in our double mutant AMLs further substantiates our model as a powerful tool to study human leukemogenesis, as these events also spontaneously occur in FLT3/ITD+ patients. This model is a powerful tool that allows us to characterize the interplay between FLT3-ITD and DNMT3A mutations in the development and maintenance of both myeloid and lymphoid leukemias, and provides a novel clinicallyrelevant model for the development and evaluation of therapies for acute leukemia.

\section{MATERIALS AND METHODS}

\section{Mice}

Mice harboring floxed Dnmt3a alleles (Dnmt $3 a^{f / f}$ ) and a pIpC-inducible Mx1-Cre were bred to a substrain of our Flt $3^{I T D /+}$ knock-in mice which retain a floxed PGKNeo cassette from the initial targeting (Figure 1A).[4, 14] Dnmt3a knock-out, and excision of the PGK-Neo cassette within the Flt $3^{I T D /+}$ allele, were achieved by two intraperitoneal injection of $\mathrm{pIpC}(250 \mathrm{ug} / \mathrm{mouse}$ in PBS; Invivogen) every other day. Genomic DNA was prepared from peripheral blood eight weeks after pIpC injection to confirm loxP recombination at both alleles (Figure S1) Primers used for genotyping and confirmation of loxP recombination (Flox F \& Flox R) can be found in Table S1. Diagnoses and classification of hematopoietic neoplasms were made based on the previously established Bethesda proposals.[39, 40] All animal experiments were performed according to protocols approved by the Animal 
Care and Use Committee of Johns Hopkins University in accordance with guidelines set forth by the National Institutes of Health.

\section{Complete peripheral blood cell count and cytology}

Mice were monitored and sacrificed when they exhibited signs of disease development (lethargy, ruffled coat, abnormal complete blood count (CBC) differential). Peripheral blood was collected from the facial vein and subjected to complete blood cell counting, and a WBC differential was performed using the Hemavet950 system (Drew Scientific). Bone marrow cytospins and peripheral blood smears were stained using a modified WrightGiemsa protocol (Sigma-Aldrich), and representative images were acquired on an Olympus BX46 microscope with an Olympus DP72 camera at $\times 50$ magnification with a 0.9 aperture. Olympus cellSens Standard 1.5 image acquisition software was used.

\section{Flow cytometry}

Diagnostic flow cytometric analysis was performed as previously described.[4] Data were analyzed using FlowJo Version 9.3.3 software (TreeStar).

\section{RT-PCR and FIt3 expression analysis}

RNA was extracted from whole bone marrow, thymus, or lymphnode where appropriate using TRIzol (Invitrogen), and reverse transcribed using the iScript cDNA Synthesis Kit (Bio-Rad). Quantitative RT-PCR was performed using the iCycler iQ multicolor real-time PCR system (Bio-Rad), and transcript levels were normalized to Rps16. Primers spanning exons 16 and 17 were used to detect Flt3 expression (Table S1).

\section{Loss of heterozygosity (LOH) analysis of Flt3 allele}

Genomic DNA was extracted from tail tissue, whole bone marrow, thymus, or lymph node where appropriate, using the Wizard Genomic DNA Purification Kit (Promega). PCR amplification was performed using 50mg of genomic DNA with Flt3 genotyping primers flanking the ITD mutation (Table S1).

\section{Transplantation}

$1 \times 10^{6}$ whole bone marrow cells from leukemic mice were transplanted into sub-lethally irradiated $\mathrm{C} 57 \mathrm{Bl} / 6$ CD45.1 recipients (7.5 cGy) by tail vein injection. Blood was collected from the facial vein every 3-4 weeks for
$\mathrm{CBCs}$ and flow cytometric analysis of engraftment. Additionally, recipients were monitored for visible signs of disease development.

\section{Stem cell compartment}

Eight weeks post pIpC injection, whole bone marrow was isolated, and stem cell and progenitor populations were quantified as previously described.[18] Phenotypic definitions of these compartments are as follows: LSK: Lin ${ }^{-} \mathrm{Sca}-1^{\mathrm{hi}}$ - $-K I T^{\mathrm{hi}}$; LT-HSC: Lin $^{-} \mathrm{Sca}-1^{\mathrm{hi}} \mathrm{c}-\mathrm{KIT}^{\mathrm{hi}} \mathrm{CD} 34^{-}$ CD135-; ST-HSC: Lin $^{-}$Sca- $1^{\text {hi }}$ c-KIT ${ }^{\text {hi }}$ CD $34^{+} \mathrm{CD} 135^{-}$; MPP: $\quad$ Lin $^{-}$Sca- ${ }^{\text {hi }}$ c-KIT ${ }^{\text {hi }} \mathrm{CD} 34^{+} \mathrm{CD} 135^{+}$; CMP: Lin $^{-}$ Sca- $1^{-}$c-KIT ${ }^{\text {hi }} \mathrm{CD} 34^{+} \mathrm{CD} 16 / 32^{\text {mid. }}$ GMP： Lin $^{-} \mathrm{Sca}-1^{-}$c$\mathrm{KIT}^{\mathrm{hi}} \mathrm{CD} 34^{+} \mathrm{CD} 16 / 32^{\mathrm{hi}}$; MEP: Lin $^{-} \mathrm{Sca}-1^{-} \mathrm{c}-\mathrm{KIT}^{\mathrm{hi}} \mathrm{CD} 34^{-}$ CD16/32-.

\section{Colony formation assays}

Bone marrow was isolated from mice 8 weeks post pIpC injection, and subjected to lineage depletion using the MACS cell separation system (Miltenyi Biotec). 10,000 lineage negative bone marrow cells were plated on Methocult M3434 (Stemcell Technologies), and analyzed at day 9-11. Colonies were disaggregated, and 200 cells were re-plated and scored for subsequent secondary and tertiary colony formation. Three mice were assayed per genotype, and experiments were performed in triplicate.

\section{ACKNOWLEDGMENTS}

We would like to thank members of the Small Lab for helpful discussions, and the flow cytometry core in the Department of Oncology at Johns Hopkins University, most notably Lee Blosser and Ada Tam. J.P. designed and performed experiments, analyzed data and wrote the manuscript; D.H. designed and performed experiments and analyzed data; L.L., B.N., K.N. and A.D. performed experiments; C.G. provided mouse model and experimental guidance; and D.S. designed experiments and revised the manuscript.

\section{CONFLICTS OF INTEREST}

The authors declare no competing financial interests.

\section{GRANT SUPPORT}

The project described was supported by grants from the National Cancer Institute (CA90668, P30 CA006973), Rally Foundation (The Truth 365 grant), Commonwealth Foundation and Giant Food Pediatric Cancer Research Fund. D.S. is also supported by the Kyle Haydock Professorship. 


\section{REFERENCES}

1. Cancer Genome Atlas Research Network. Genomic and epigenomic landscapes of adult de novo acute myeloid leukemia. The New England Journal of Medicine. 2013;368:2059-2074.

2. Welch JS, Ley TJ, Link DC, Miller CA, Larson DE, Koboldt DC, Wartman LD, Lamprecht TL, Liu F, Xia J, Kandoth C, Fulton RS, McLellan MD, et al. The origin and evolution of mutations in acute myeloid leukemia. Cell. 2012;150:264-278.

3. Patel JP, Gönen M, Figueroa ME, Fernandez H, Sun Z, Racevskis J, Van Vlierberghe P, Dolgalev I, Thomas $\mathrm{S}$, Aminova O, Huberman K, Cheng J, Viale A, et al. Prognostic relevance of integrated genetic profiling in acute myeloid leukemia. The New England Journal of Medicine. 2012;366:1079-1089.

4. Li L, Piloto O, Nguyen HB, Greenberg K, Takamiya K, Racke F, Huso D, Small D. Knock-in of an internal tandem duplication mutation into murine FLT3 confers myeloproliferative disease in a mouse model. Blood. 2008;111:3849-3858.

5. Lee BH, Tothova Z, Levine RL, Anderson K, Buza-Vidas N, Cullen DE, McDowell EP, Adelsperger J, Fröhling S, Huntly BJ, Beran M, Jacobsen SE, Gilliland DG. FLT3 mutations confer enhanced proliferation and survival properties to multipotent progenitors in a murine model of chronic myelomonocytic leukemia. Cancer Cell. 2007; 12:367-380.

6. Vogelstein B, Papadopoulos N, Velculescu VE, Zhou S, Diaz LA Jr, Kinzler KW. Cancer genome landscapes. Science, 2013;339:1546-1558.

7. Ley TJ, Ding L, Walter MJ, McLellan MD, Lamprecht T, Larson DE, Kandoth C, Payton JE, Baty J, Welch J, Harris CC, Lichti CF, Townsend RR, et al. DNMT3A mutations in acute myeloid leukemia. The New England Journal of Medicine. 2010;363:2424-2433.

8. Yang L, Rau R, Goodell MA. DNMT3A in haematological malignancies. Nature Reviews.Cancer. 2015;15:152-165.

9. Russler-Germain DA, Spencer DH, Young MA, Lamprecht TL, Miller CA, Fulton R, Meyer MR, Erdmann-Gilmore P, Townsend RR, Wilson RK, Ley TJ. The R882H DNMT3A mutation associated with AML dominantly inhibits wildtype DNMT3A by blocking its ability to form active tetramers. Cancer Cell. 2014;25:442-454.

10. Kim SJ, Zhao H, Hardikar S, Singh AK, Goodell MA, Chen T. A DNMT3A mutation common in AML exhibits dominant-negative effects in murine ES cells. Blood. 2013;122:4086-4089.

11. Holz-Schietinger C, Matje DM, Reich NO. Mutations in DNA methyltransferase (DNMT3A) observed in acute myeloid leukemia patients disrupt processive methylation. The Journal of Biological Chemistry. 2012;287:3094130951.
12. Mayle A, Yang L, Rodriguez B, Zhou T, Chang E, Curry CV, Challen GA, Li W, Wheeler D, Rebel VI, Goodell MA. Dnmt3a loss predisposes murine hematopoietic stem cells to malignant transformation. Blood. 2015;125:629-638.

13. Challen GA, Sun D, Jeong M, Luo M, Jelinek J, Berg JS, Bock C, Vasanthakumar A, Gu H, Xi Y, Liang S, Lu Y, Darlington GJ, et al. Dnmt3a is essential for hematopoietic stem cell differentiation. Nature Genetics. 2011;44:23-31.

14. Kaneda M, Okano M, Hata K, Sado T, Tsujimoto N, Li E, Sasaki H. Essential role for de novo DNA methyltransferase Dnmt3a in paternal and maternal imprinting. Nature. 2004;429:900-903.

15. Jeong M, Sun D, Luo M, Huang Y, Challen GA, Rodriguez B, Zhang X, Chavez L, Wang H, Hannah R, Kim SB, Yang L, Ko M, et al. Large conserved domains of low DNA methylation maintained by Dnmt3a. Nature Genetics. 2014;46:17-23.

16. Xie M, Lu C, Wang J, McLellan MD, Johnson KJ, Wendl MC, McMichael JF, Schmidt HK, Yellapantula V, Miller CA, Ozenberger BA, Welch JS, Link DC, et al. Age-related mutations associated with clonal hematopoietic expansion and malignancies. Nature Medicine. 2014;20:1472-1478.

17. Shlush LI, Zandi S, Mitchell A, Chen WC, Brandwein JM, Gupta V, Kennedy JA, Schimmer AD, Schuh AC, Yee KW, McLeod JL, Doedens M, Medeiros JJ, et al. Identification of pre-leukaemic haematopoietic stem cells in acute leukaemia. Nature. 2014;506:328-333.

18. Chu SH, Heiser D, Li L, Kaplan I, Collector M, Huso D, Sharkis SJ, Civin C, Small D. FLT3-ITD knockin impairs hematopoietic stem cell quiescence/homeostasis, leading to myeloproliferative neoplasm. Cell Stem Cell. 2012;11:346358.

19. Kühn R, Schwenk F, Aguet M, Rajewsky K. Inducible gene targeting in mice. Science. 1995;269:1427-1429.

20. Greenblatt S, Li L, Slape C, Nguyen B, Novak R, Duffield A, Huso D, Desiderio S, Borowitz MJ, Aplan P, Small D. Knock-in of a FLT3/ITD mutation cooperates with a NUP98-HOXD13 fusion to generate acute myeloid leukemia in a mouse model. Blood. 2012;119:2883-2894.

21. Bailey E, Li L, Duffield AS, Ma HS, Huso DL, Small D. FLT3/D835Y mutation knock-in mice display less aggressive disease compared with FLT3/internal tandem duplication (ITD) mice. Proceedings of the National Academy of Sciences of the United States of America. 2013;110:21113-21118.

22. Meshinchi S, Alonzo TA, Stirewalt DL, Zwaan M, Zimmerman M, Reinhardt D, Kaspers GJ, Heerema NA, Gerbing R, Lange BJ, Radich JP. Clinical implications of FLT3 mutations in pediatric AML. Blood. 2006;108:36543661 .

23. Whitman SP, Archer KJ, Feng L, Baldus C, Becknell B, Carlson BD, Carroll AJ, Mrózek K, Vardiman JW, George SL, Kolitz JE, Larson RA, Bloomfield CD, et al. Absence of the wild-type allele predicts poor prognosis in adult de 
novo acute myeloid leukemia with normal cytogenetics and the internal tandem duplication of FLT3: A cancer and leukemia group B study. Cancer Research. 2001;61:72337239 .

24. Thiede C, Steudel C, Mohr B, Schaich M, Schäkel U, Platzbecker U, Wermke M, Bornhäuser M, Ritter M, Neubauer A, Ehninger G, Illmer T. Analysis of FLT3activating mutations in 979 patients with acute myelogenous leukemia: Association with FAB subtypes and identification of subgroups with poor prognosis. Blood. 2002;99:43264335 .

25. Rau R, Magoon D, Greenblatt S, Li L, Annesley C, Duffield AS, Huso D, McIntyre E, Clohessy JG, Reschke M, Pandolfi PP, Small D, Brown P. NPMc+ cooperates with FLT3/ITD mutations to cause acute leukemia recapitulating human disease. Experimental Hematology. 2014;42:101-13. e5.

26. Neumann M, Heesch S, Gökbuget N, Schwartz S, Schlee C, Benlasfer O, Farhadi-Sartangi N, Thibaut J, Burmeister T, Hoelzer D, Hofmann WK, Thiel E, Baldus CD. Clinical and molecular characterization of early $\mathrm{T}$-cell precursor leukemia: A high-risk subgroup in adult T-ALL with a high frequency of FLT3 mutations. Blood Cancer Journal. 2012;2:e55.

27. Neumann M, Heesch S, Schlee C, Schwartz S, Gökbuget N, Hoelzer D, Konstandin NP, Ksienzyk B, Vosberg S, Graf A, Krebs S, Blum H, Raff T, et al. Whole-exome sequencing in adult ETP-ALL reveals a high rate of DNMT3A mutations. Blood. 2013;121:4749-4752.

28. Grossmann V, Haferlach C, Weissmann S, Roller A, Schindela S, Poetzinger F, Stadler K, Bellos F, Kern W, Haferlach T, Schnittger S, Kohlmann A. The molecular profile of adult T-cell acute lymphoblastic leukemia: Mutations in RUNX1 and DNMT3A are associated with poor prognosis in T-ALL. Genes, Chromosomes \& Cancer. 2013;52:410-422.

29. De Obaldia ME, Bell JJ, Wang X, Harly C, YashiroOhtani Y, DeLong JH, Zlotoff DA, Sultana DA, Pear WS, Bhandoola A. T cell development requires constraint of the myeloid regulator $\mathrm{C} / \mathrm{EBP}$-alpha by the notch target and transcriptional repressor Hes1. Nature Immunology. 2013;14:1277-1284.

30. Li Z, Cai X, Cai CL, Wang J, Zhang W, Petersen BE, Yang $\mathrm{FC}, \mathrm{Xu}$ M. Deletion of Tet2 in mice leads to dysregulated hematopoietic stem cells and subsequent development of myeloid malignancies. Blood. 2011;118:4509-4518.

31. Moran-Crusio K, Reavie L, Shih A, Abdel-Wahab O, Ndiaye-Lobry D, Lobry C, Figueroa ME, Vasanthakumar A, Patel J, Zhao X, Perna F, Pandey S, Madzo J, et al. Tet2 loss leads to increased hematopoietic stem cell self-renewal and myeloid transformation. Cancer Cell. 2011;20:11-24.
32. Chan G, Kalaitzidis D, Usenko T, Kutok JL, Yang W, Mohi MG, Neel BG. Leukemogenic Ptpn11 causes fatal myeloproliferative disorder via cell-autonomous effects on multiple stages of hematopoiesis. Blood. 2009;113:44144424.

33. Jan M, Snyder TM, Corces-Zimmerman MR, Vyas P, Weissman IL, Quake SR, Majeti R. Clonal evolution of preleukemic hematopoietic stem cells precedes human acute myeloid leukemia. Sci Transl Med. 2012;4:49ra118.

34. Corces-Zimmerman MR, Hong WJ, Weissman IL, Medeiros BC, Majeti R. Preleukemic mutations in human acute myeloid leukemia affect epigenetic regulators and persist in remission. Proc Natl Acad Sci U S A. 2014;111:2548-2553.

35. Meyer SE, Qin $T$, Muench DE, Masuda $K$, Venkatasubramanian M, Orr E, Suarez L, Gore SD, Delwel R, Paietta E, Tallman MS, Fernandez H, Melnick A, et al. DNMT3A Haploinsufficiency Transforms FLT3ITD Myeloproliferative Disease into a Rapid, Spontaneous, and Fully Penetrant Acute Myeloid Leukemia. Cancer Discov. 2016;6:501-515.

36. Yang L, Rodriguez B, Mayle A, Park HJ, Lin X, Luo M, Jeong M, Curry CV, Kim SB, Ruau D, Zhang X, Zhou T, Zhou M, et al. DNMT3A Loss Drives Enhancer Hypomethylation in FLT3-ITD-Associated Leukemias. Cancer Cell. 2016;29:922-934.

37. Paietta E, Ferrando AA, Neuberg D, Bennett JM, Racevskis J, Lazarus H, Dewald G, Rowe JM, Wiernik PH, Tallman MS, Look AT. Activating FLT3 mutations in CD117/ KIT(+) T-cell acute lymphoblastic leukemias. Blood. 2004;104:558-560.

38. Grossmann V, Haferlach C, Weissmann S, Roller A, Schindela S, Poetzinger F, Stadler K, Bellos F, Kern W, Haferlach T, Schnittger S, Kohlmann A. The molecular profile of adult T-cell acute lymphoblastic leukemia: Mutations in RUNX1 and DNMT3A are associated with poor prognosis in T-ALL. Genes, Chromosomes \& Cancer. 2013;52:410-422.

39. Kogan SC, Ward JM, Anver MR, Berman JJ, Brayton C, Cardiff RD, Carter JS, de Coronado S, Downing JR, Fredrickson TN, Haines DC, Harris AW, Harris NL, et al. Bethesda proposals for classification of nonlymphoid hematopoietic neoplasms in mice. Blood. 2002;100:238245.

40. Morse HC 3rd, Anver MR, Fredrickson TN, Haines DC, Harris AW, Harris NL, Jaffe ES, Kogan SC, MacLennan IC, Pattengale PK, Ward JM; Hematopathology subcommittee of the Mouse Models of Human Cancers Consortium. Bethesda proposals for classification of lymphoid neoplasms in mice. Blood. 2002;100:246-258. 